\title{
On the Complexity of Various Parameterizations of Common Induced Subgraph Isomorphism*
}

\author{
Faisal N. Abu-Khzam ${ }^{\dagger} \quad$ Édouard Bonnet ${ }^{\ddagger} \quad$ Florian Sikora ${ }^{\S}$
}

\begin{abstract}
In the MAXimum COMmon InduCED SubGRAPH problem (henceforth MCIS), given two graphs $G_{1}$ and $G_{2}$, one looks for a graph with the maximum number of vertices being both an induced subgraph of $G_{1}$ and $G_{2}$. MCIS is among the most studied classical NP-hard problems. It remains NP-hard on many graph classes including forests. In this paper, we study the parameterized complexity of MCIS. As a generalization of CLIQUE, it is W[1]-hard parameterized by the size of the solution. Being NP-hard even on forests, most structural parameterizations are intractable. One has to go as far as parameterizing by the size of the minimum vertex cover to get some tractability. Indeed, when parameterized by $k:=$ $\operatorname{vc}\left(G_{1}\right)+\operatorname{vc}\left(G_{2}\right)$ the sum of the vertex cover number of the two input graphs, the problem was shown to be fixed-parameter tractable, with an algorithm running in time $2^{O(k \log k)}$. We complement this result by showing that, unless the ETH fails, it cannot be solved in time $2^{o(k \log k)}$. This kind of tight lower bound has been shown for a few problems and parameters but, to the best of our knowledge, not for the vertex cover number. We also show that MCIS does not have a polynomial kernel when parameterized by $k$, unless NP $\subseteq$ coNP/poly. Finally, we study MCIS and its connected variant MCCIS on some special graph classes and with respect to other structural parameters.
\end{abstract}

\section{Introduction}

A common induced subgraph of two graphs $G_{1}$ and $G_{2}$ is a graph that is isomorphic to an induced subgraph of both graphs. The problem of finding a common induced subgraph with the maximum number of vertices (or edges) has many applications in a number of domains including bioinformatics and chemistry $[17,21,25,29,30]$. In the decision version of the problem, we are given an integer $k$ and the question is to decide whether there is a solution with at least $k$ vertices. We say that the solution size $k$ is the natural parameter of the problem.

Concerning its classical complexity, Maximum Common Induced SubGraph is NP-complete, and remains so on forests. When the common subgraph is required to be connected, the problem is in $\mathrm{P}$ for trees [16]. Moreover, Maximum Common Induced Subgraph is also in $\mathrm{P}$ when the two input graphs are connected and (both) have bounded treewidth and bounded degree [4].

A particular case of Maximum Common Induced Subgraph is the well known Induced Subgraph Isomorphism (ISI) decision problem, where the question posed is whether $G_{1}$ is isomorphic to an induced subgraph of $G_{2}$. In other words, it is equivalent to Maximum Common INDUCED SUBGRAPH where $k=\left|G_{1}\right|$. In this case, $G_{1}$ is called the pattern graph and $G_{2}$ is called the host graph. ISI is known to be NP-hard, even when $G_{2}$ is an interval graph and $G_{1}$ is a proper interval graph, but it becomes polynomial-time solvable when $G_{1}$ is in addition

\footnotetext{
*An extended abstract of this work appears in [2]

${ }^{\dagger}$ Lebanese American University, Beirut, Lebanon faisal.abukhzam@lau.edu.lb

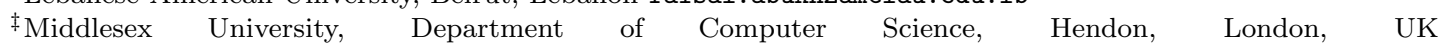
edouard.bonnet@lamsade.dauphine.fr

§Université Paris-Dauphine, PSL Research University, CNRS, LAMSADE, Paris, France, florian.sikora@dauphine.fr
} 
connected [19]. Unlike Subgraph Isomorphism, Induced Subgraph Isomorphism remains NP-hard when both the host graph and the pattern graph consist of a disjoint union of paths [11]. From the parameterized complexity viewpoint, the problem is $\mathrm{W}[1]$-hard in general for the natural parameter, by a straightforward reduction from $k$-CLIQUE. Therefore MCIS is also W[1]-hard. Moreover, ISI (and, therefore, MCIS) remains W[1]-hard even when both graphs are interval graphs [24]. On the other hand, ISI is FPT on nowhere dense graphs, being expressible by a first-order formula of length function of the natural parameter $k$ [18]. This generalizes what was previously known about ISI on $H$-minor free graphs [15] and graphs of bounded degree [8]. We observe that whenever ISI in FPT on a certain graph class, then so is MCIS. To see this, note that an arbitrary instance $\left(G_{1}, G_{2}, k\right)$ of MCIS can be reduced in fpt-time to instances of ISI by enumerating each graph $H$ on $k$ vertices and checking whether $H$ is an induced subgraph of $G_{1}$ and $G_{2}$. This implies that ISI and MCIS have the same parameterized complexity with respect to the natural parameter.

Another way of dealing with the hardness of a problem is to study its complexity with respect to auxiliary (or structural) parameters, to better understand its algorithmic behavior (see for example [13]). Being NP-hard on disjoint union of chordless paths [11], MCIS is hard on graphs with bounded treewidth as well as graphs where the size of the minimum feedback vertex set is bounded. Thus the problem is paraNP-hard when parameterized by the treewidth of the input graphs, or by a bound on the sizes of their minimum feedback vertex sets. Therefore, we need to look for "bigger" parameters. And indeed, if the parameter $k$ is a bound on the sizes of the minimum vertex covers of the input graphs, then the problem is in FPT, with a running time of $O\left((24 k)^{k}\right)=2^{O(k \log k)}$ [1]. In this paper, we show that this algorithm cannot be significantly improved: unless the Exponential Time Hypothesis (ETH) fails, there is no algorithm solving MCIS in time $O^{*}\left(2^{o(k \log k)}\right)$, where the $O^{*}$ notation suppresses the polynomial factors. We also prove that MCIS does not have a polynomial-size kernel in this case unless NP $\subseteq$ coNP/poly. These two latter results answer open problems raised in [1]. Finally, we show that MAximum Common Connected Induced SubGraph (MCCIS), where the solution should be a connected graph, is also fixed-parameter tractable when parameterized by $k:=v c\left(G_{1}\right)+v c\left(G_{2}\right)$.

\section{Preliminaries}

Two finite graphs $G_{1}=\left(V_{1}, E_{1}\right)$ and $G_{2}=\left(V_{2}, E_{2}\right)$ are isomorphic if there is a bijection $\pi$ : $V_{1} \rightarrow V_{2}$ such that $\forall u, v \in V_{1}: u v \in E_{1} \Leftrightarrow \pi(u) \pi(v) \in E_{2}$. Given a graph $G=(V, E)$, a graph $G^{\prime}=\left(V^{\prime}, E^{\prime}\right)$ is an induced subgraph of $G$ if $V^{\prime} \subseteq V$ and $E^{\prime}=E\left(V^{\prime}\right)=\left\{u v \in E \mid u, v \in V^{\prime}\right\}$, i.e. $E^{\prime}$ is the edge set with both extremities in $V^{\prime}$. We also say that $G^{\prime}$ is the subgraph of $G$ induced by $V^{\prime}$.

The girth of a graph $G$ is the length of the shortest cycle contained in $G$. Contracting an edge $u v$ consists of deleting $u v$ and replacing the vertices $u$ and $v$ by a single vertex $w$ in the incidence relation (edges incident on $u$ or $v$ become incident on $w$ ). A graph $H$ is a minor of graph $G$ if $H$ is obtained from a subgraph of $G$ by applying zero or more edge contractions. Given a fixed graph $H$, a family $\mathcal{F}$ of graphs is said to be $H$-minor free if $H$ is not a minor of any element of $\mathcal{F}$.

The Maximum Common Induced Subgraph problem is defined formally as follows.

Maximum Common InduCed SubGraph (MCIS):

- Input: Two graphs $G_{1}=\left(V_{1}, E_{1}\right)$ and $G_{2}=\left(V_{2}, E_{2}\right)$.

- Output: An induced subgraph $G_{1}^{\prime}$ of $G_{1}$ isomorphic to an induced subgraph $G_{2}^{\prime}$ of $G_{2}$ with

a maximum number of vertices.

Maximum Common Connected Induced Subgraph (MCCIS) is defined as MCIS with the additional restriction that the solution must be connected.

For completeness, we also give the definition of Induced SubGraph Isomorphism: 
INDUCED SUbGRAPH ISOMORPHISM (ISI):

- Input: Two graphs $G_{1}=\left(V_{1}, E_{1}\right)$ and $G_{2}=\left(V_{2}, E_{2}\right)$.

- Output: An induced subgraph $G_{2}^{\prime}$ of $G_{2}$ isomorphic to $G_{1}$ if it exists.

Induced Connected Subgraph Isomorphism (ICSI) is defined as ISI but $G_{1}$ must be connected.

Parameterized complexity A parameterized problem $(I, k)$ is fixed-parameter tractable (or in the class FPT) with respect to parameter $k$ if it can be solved in $f(k) \cdot|I|^{c}$ time (i.e. in fpttime), where $f$ is any computable function and $c$ is a constant (see $[12,27]$ for more details about fixed-parameter tractability). The parameterized complexity hierarchy is composed of the classes $\mathrm{FPT} \subseteq \mathrm{W}[1] \subseteq \mathrm{W}[2] \subseteq \cdots \subseteq \mathrm{XP}$. The class $\mathrm{XP}$ contains problems solvable in time $f(k) \cdot \mid I^{g(k)}$, where $f$ and $g$ are unrestricted functions. A problem is said to be paraNP-hard if it is NP-hard even for a constant value of the parameter (it hence cannot be in XP). A W[1]-hard problem is not fixed-parameter tractable, unless FPT $=\mathrm{W}[1]$, and one can prove $\mathrm{W}[1]$-hardness by means of a parameterized reduction from a W[1]-hard problem. This is a mapping of an instance $(I, k)$ of a problem $A_{1}$ in $g(k) \cdot|I|^{O(1)}$ time (for any computable function $g$ ) into an instance $\left(I^{\prime}, k^{\prime}\right)$ for $A_{2}$ such that $(I, k) \in A_{1} \Leftrightarrow\left(I^{\prime}, k^{\prime}\right) \in A_{2}$ and $k^{\prime} \leq h(k)$ for some function $h$.

A powerful technique to design parameterized algorithms is kernelization. In short, kernelization is a polynomial-time self-reduction algorithm that takes an instance $(I, k)$ of a parameterized problem $P$ as input and computes an equivalent instance $\left(I^{\prime}, k^{\prime}\right)$ of $P$ such that $\left|I^{\prime}\right| \leqslant h(k)$ for some computable function $h$ and $k^{\prime} \leqslant k$. The instance $\left(I^{\prime}, k^{\prime}\right)$ is called a kernel in this case. If the function $h$ is polynomial, we say that $\left(I^{\prime}, k^{\prime}\right)$ is a polynomial kernel. It is well known that a problem is in FPT iff it has a kernel, but this equivalence yields super-polynomial kernels (in general). To design efficient parameterized algorithms, a kernel of polynomial (or even linear) size in $k$ is important. However, some lower bounds on the size of the kernel can be shown unless some polynomial hierarchy collapses. To show this result, we will use the cross composition technique developed by Bodlaender et al. [7].

Definition 1 (Polynomial equivalence relation [7]). An equivalence relation $\mathcal{R}$ on $\Sigma^{*}$ is said to be polynomial if the following two conditions hold: (i) There is an algorithm that given two strings $x, y \in \Sigma^{*}$ decides whether $x$ and $y$ belong to the same equivalence class in time $(|x|+|y|)^{O(1)}$. (ii) For any finite set $S \subseteq \Sigma^{*}$ the equivalence relation $\mathcal{R}$ partitions the elements of $S$ into at most $\left(\max _{x \in S}|x|\right)^{O(1)}$ classes.

Definition 2 (OR-cross-composition [7]). Let $L \subseteq \Sigma^{*}$ be a set and let $Q \subseteq \Sigma^{*} \times \mathbb{N}$ be a parameterized problem. We say that $L$ cross-composes into $Q$ if there is a polynomial equivalence relation $\mathcal{R}$ and an algorithm which, given $t$ strings $x_{1}, x_{2}, \ldots, x_{t}$ belonging to the same equivalence class of $\mathcal{R}$, computes an instance $\left(x^{*}, k^{*}\right) \in \Sigma^{*} \times \mathbb{N}$ in time polynomial in $\sum_{i=1}^{t}\left|x_{i}\right|$ such that: (i) $\left(x^{*}, k^{*}\right) \in Q \Leftrightarrow x_{i} \in L$ for some $1 \leqslant i \leqslant t$. (ii) $k^{*}$ is bounded by a polynomial in $\max _{i=1}^{t}\left|x_{i}\right|+\log t$.

Proposition 3 ([7]). Let $L \subseteq \Sigma^{*}$ be a set which is NP-hard under Karp reductions. If $L$ cross-composes into the parameterized problem $Q$, then $Q$ has no polynomial kernel unless NP $\subseteq$ coNP/poly.

The Exponential Time Hypothesis (ETH) is a conjecture by Impagliazzo et al. asserting that there is no $2^{o(n)}$-time algorithm for 3-SAT on instances with $n$ variables [20]. The ETH, together with the sparsification lemma [20], even implies that there is no $2^{o(n+m)}$-time algorithm solving 3-SAT. Many algorithmic lower bounds have been proved under the ETH, see for example [22].

We say that a parameterized problem is fixed-parameter enumerable if all feasible solutions can be enumerated in $O\left(f(k)|I|^{c}\right)$ time, where $f$ is a computable function of the parameter $k$ only, and $c$ is a constant. 


\section{Parameterized Complexity with respect to the natural parameter}

We study the parameterized complexity of Induced Subgraph Isomorphism, Maximum Common Induced Subgraph, Induced Connected Subgraph Isomorphism, and Maximum Common Connected Induced Subgraph with respect to the natural parameter. We will in particular study these problems in graphs of bounded degeneracy, chordal graphs, and graphs of large girth.

Theorem 4. MCIS, MCCIS, ISI, and ICSI are W[1]-complete.

Proof. Since those problems are W[1]-hard by a straightforward reduction from $k$-CLIQUE, it suffices to show membership in W[1]. In [9], it is shown that if a problem can be reduced in FPT time to simulating a non-deterministic single-taped Turing machine halting in at most $f(k)$ steps, for some function $f$, then it is in $\mathrm{W}[1]$. The Turing machine can have an alphabet and a set of states of size depending on the size of the input of the initial problem. In our case, we can design a Turing machine that guesses in $2 k$ steps the corresponding right $k$ vertices in $G_{1}$ (for $\mathrm{I}(\mathrm{C}) \mathrm{SI}$ this part is not necessary) and the right $k$ vertices in $G_{2}$ (our alphabet being isomorphic to an indexing of $\left.V\left(G_{1}\right) \cup V\left(G_{2}\right)\right)$ and then check in time $O\left(k^{2}\right)$ whether the two induced subgraphs are isomorphic (and that they are connected for ICSI and MCCIS).

In [26] it was shown that Maximum Induced MATChing ${ }^{1}$ is W[1]-hard on bipartite graphs. This implies that MCIS is W[1]-hard on bipartite graphs. In fact, we show that MCIS remains W[1]-hard on more restricted graph classes, namely $C_{4}$-free bipartite graphs with degeneracy 2 . In particular, those graphs have girth at least 6 . This result tells us two things about MC(C)IS. The first is that the fixed-parameter algorithm of Cai et al. [8, Theorem 1] cannot be extended from bounded degree to bounded degeneracy (note that some W-hard problems on general graphs become FPT on graphs with bounded degeneracy, such as the W[2]-complete Dominating SET problem [5]). The second is that short cycles are not making MC(C)IS W[1]-hard; they are W[1]hard even without them. In [28], the authors present fixed-parameter algorithms on graphs of girth 5 , for some problems which are W-hard on general graphs. MCIS and MCCIS are also resistant to this approach.

Theorem 5. Induced Subgraph Isomorphism and Induced Connected Subgraph IsoMORPHISM are $\mathrm{W}[1]$-complete even when both graphs are $C_{4}$-free bipartite graphs with degeneracy at most 2 .

Proof. The incidence graph $I(G)$ of any graph $G=(V, E)$, obtained by subdividing each edge of $G$ once, has degeneracy 2. Indeed, graph $I(G)$ is the bipartite graph $(V \uplus E, F)$ where the edges of $F$ are all the $u e$ for which $u \in V, e \in E$, and $u$ is an endpoint of $e$. All the vertices $e \in E$ of $I(G)$ have degree 2. Therefore, they can be removed first. Then, what is left in $I(G)$ is the independent set $V$.

We transform any input $G=(V, E), k>3$ of $k$-CLIQUE, into the instance $I\left(K_{k}\right), I(G)$ of $\mathrm{I}(\mathrm{C}) \mathrm{SI}$, where both graphs have degeneracy 2. The problem consists of finding the incidence graph of a $k$-clique within the incidence graph of $G$. We show that it is equivalent to finding a $k$-clique in $G$. Obviously, if there is a $k$-clique $S$ in $G$, then the graph $I(G)[S \cup E(S)]$ is isomorphic to $I\left(K_{k}\right)$. Now, let us assume that $I\left(K_{k}\right)$ is isomorphic to an induced subgraph of $I(G)$. We denote by $a_{1}, \ldots, a_{k}$ the vertices of $I\left(K_{k}\right)$ with degree $k-1$, and by $b_{1}, \ldots, b_{\left(\begin{array}{c}k \\ 2\end{array}\right)}$ the vertices of $I\left(K_{k}\right)$ with degree 2 . We denote by $\psi: V\left(I\left(K_{k}\right)\right) \rightarrow V(I(G))$ the isomorphism from graph $I\left(K_{k}\right)$ to an induced subgraph of $I(G)$. Let $u_{i}=\psi\left(a_{i}\right)$ for each $i \in[k]$, and $v_{j}=\psi\left(b_{j}\right)$ for each $j \in\left[\left(\begin{array}{l}k \\ 2\end{array}\right)\right]$. We set $S=\left\{u_{1}, \ldots, u_{k}, v_{1}, \ldots, v_{\left(\begin{array}{c}k \\ 2\end{array}\right)}\right\}$. For every $i \in[k], u_{i} \in V$ since the degree of $a_{i}$ in $I\left(K_{k}\right)$ is $k-1>2$ (hence, the degree of $u_{i}$ in $S$ is also $k-1>2$ ). Now, for every $j \in\left[\left(\begin{array}{c}k \\ 2\end{array}\right)\right], v_{j} \in E$ since

\footnotetext{
${ }^{1}$ where one looks for a largest subset of vertices that induce a disjoint union of edges
} 
$v_{j}$ has two neighbors in $V$ (recall that $I(G)$ is bipartite). Therefore, $u_{1}, \ldots, u_{k}$ are $k$ vertices in $V$ inducing precisely $\left(\begin{array}{c}k \\ 2\end{array}\right)$ edges. Hence, $\left\{u_{1}, \ldots, u_{k}\right\}$ is a $k$-clique in $G$.

Membership in W[1] comes from Theorem 4.

Corollary 6. Maximum Common Induced Subgraph and Maximum Common Connected INDUCED SUBGRAPH remain W[1]-complete on bipartite graphs of girth 6 and degeneracy 2.

The absence of triangles and cycles of length four in the input graphs does not make the problems tractable. We show that the absence of a long induced cycle does not help either (in [6], the authors show that the W[2]-hard problem Dominator Coloring is in FPT when the input graph is chordal). More specifically, all four problems are $\mathrm{W}[1]$-hard on chordal graphs. In fact, we can even show that these problems remain $\mathrm{W}[1]$-hard on a proper subclass of chordal graphs called split graphs. A split graph is a graph whose vertex set can be partitioned into a set inducing a clique and an independent set.

Theorem 7. ISI (hence MCIS) and ICSI (hence MCCIS) remain W[1]-hard on split graphs.

Proof. Similarly to the previous construction, we define $I^{\prime}(G)$ as the graph $(V \uplus E, F)$ where the edges of $F$ are the edges $u e$ for which $u \in V, e \in E$, and $u$ is an endpoint of $e$, plus all the edges $u v$ with $u, v \in V$. The graph $I^{\prime}(G)$ is split: $V$ induces a clique in $I^{\prime}(G)$ and $E$ induces an independent set. From an instance $G$ of $k$-CLIQUE with $k>3$, we build the equivalent instance $I^{\prime}\left(K_{k}\right), I^{\prime}(G)$ of $\mathrm{MC}(\mathrm{C}) \mathrm{IS}$ and $\mathrm{I}(\mathrm{C}) \mathrm{SI}$. The soundness can be obtained in the same way as in the previous proof.

Let us now say some words about the complexity of the connected version. First we note that MCIS is NP-hard on forests while MCCIS is solvable in polynomial-time in this case: given two forests $G_{1}$ and $G_{2}$, run the polynomial-time MCIS algorithm of Akutsu on every pair of trees from $G_{1}$ and $G_{2}$ [3]. From the parameterized complexity standpoint, MAXIMUM Common Connected Induced Subgraph is FPT whenever Induced Subgraph Isomorphism is FPT since the enumeration of all $O\left(2^{k^{2}}\right)$ possible induced connected subgraphs can be used as described in the introduction. The converse is true on classes of graphs which are closed by adding a universal vertex (i.e., a vertex linked to all the other vertices). An instance $\left(G_{1}, G_{2}, k\right)$ of ISI can be reduced to an equivalent instance $\left(G_{1}^{\prime}, G_{2}^{\prime}, k+1\right)$ of MCCIS by letting $G_{i}^{\prime}$ be the graph obtained by adding a vertex to $G_{i}$ that is made adjacent to all other vertices of $G_{i}$.

\section{Structural parameterization}

Let us first recall that $\operatorname{tw}(G) \leqslant \operatorname{fvs}(G)+1 \leqslant \operatorname{vc}(G)+1$, where $\operatorname{tw}(G)$ (resp. fvs $(G)$, $\operatorname{vc}(G)$ ) represents the treewidth (resp. the feedback vertex set number, the vertex cover number) of $G$ [14]. As noted before, if the parameter is the combination of $\operatorname{tw}\left(G_{1}\right)$ and $\operatorname{tw}\left(G_{2}\right)$ then MCIS is known to be W[1]-hard. Even more, if the parameter is the combination of $\operatorname{fvs}\left(G_{1}\right)$ and $\operatorname{fvs}\left(G_{2}\right)$ (which is bigger than the combination of the treewidth), then the problem is not even in XP since Maximum Common Induced Subgraph and Induced Subgraph Isomorphism are NP-hard on disjoint union of chordless paths, a case where the parameter is equal to $0[11,16]$.

Theorem 8 ([11, 16]). Maximum Common Induced Subgraph is paraNP-hard when parameterized by fvs $\left(G_{1}\right)+f v s\left(G_{2}\right)$ (and hence by $t w\left(G_{1}\right)+t w\left(G_{2}\right)$ ).

One can extend this result to make it valid for the connected version.

Theorem 9. Induced Connected Subgraph Isomorphism, and as a corollary Maximum Common Connected Induced Subgraph, are paraNP-hard when parameterized by fvs $\left(G_{1}\right)+$ fvs $\left(G_{2}\right)$.

Proof. Given an instance of Induced Subgraph Isomorphism on forests $G_{1}$ and $G_{2}$ (each with at least 2 trees), we build an instance of Induced Connected Subgraph Isomorphism by 
adding a universal vertex (connected to every node) in $G_{1}$ and in $G_{2}$. Both graph have thus a feedback vertex set of value one. One can see that these two universal vertices must be matched together since they are the only ones with sufficiently high degree. Then, there is a solution for Induced Subgraph Isomorphism iff there is a solution for Induced ConneCted Subgraph IsOMORPHISM. The result of course holds for MCCIS, too.

It was shown in [1] that MCIS is in FPT if the parameter is the combination of $\operatorname{vc}\left(G_{1}\right)$ and $\operatorname{vc}\left(G_{2}\right)$. Accordingly, the problem has a kernel, but no polynomial bound is known on its size. We show that, in this case, the kernel cannot be polynomial unless NP $\subseteq$ coNP/poly.

Theorem 10. Unless NP $\subseteq$ coNP/poly, Maximum Common Induced Subgraph has no polynomial kernel when parameterized by the sum of the sizes of vertex covers in the two input graphs.

Proof. We will define an OR-cross-composition from the NP-complete CLIQUE, problem, where the given instance is a tuple $\left(G^{c}, l\right)$ and the question is whether the graph $G^{c}$ contains a clique on $l$ vertices.

Given $t$ instances, $\left(G_{1}^{c}, l_{1}\right),\left(G_{2}^{c}, l_{2}\right), \ldots,\left(G_{t}^{c}, l_{t}\right)$, of Clique, where $G_{i}^{c}$ is a graph and $l_{i} \in$ $\mathbb{N}, \forall 1 \leqslant i \leqslant t$, we define our equivalence relation $\mathcal{R}$ such that any strings that are not encoding valid instances are equivalent, and $\left(G_{i}^{c}, l_{i}\right),\left(G_{j}^{c}, l_{j}\right)$ are equivalent iff $\left|V\left(G_{i}^{c}\right)\right|=\left|V\left(G_{j}^{c}\right)\right|$, and $l_{i}=l_{j}$. Hereafter, we assume that $V\left(G_{i}^{c}\right)=\{1, \ldots, n\}$ and $l_{i}=l$, for any $1 \leqslant i \leqslant t$. We will build an instance of MAXImum COMMON INDUCED SUbGRAPH parameterized by the vertex cover $\left(G_{1}, G_{2}, l^{\prime}, Z\right)$ where $G_{1}$ and $G_{2}$ are two graphs, $l^{\prime} \in \mathbb{N}$ and $Z \subseteq V\left(G_{2}\right)$ is a vertex cover of $G_{2}$ computed in fpt-time, such that there is a solution of size $l^{\prime}$ for MAXimum Common Induced SUBGRAPH iff there is an $i, 1 \leqslant i \leqslant t$ such that there is a solution of size $l$ in $G_{i}^{c}$. We will now describe how to build $G_{1}$ and $G_{2}$.

To build $G_{2}$ (see also Figure 1):

- $V\left(G_{2}\right)=\{p, q, r\} \cup\left\{a_{i} \mid 1 \leqslant i \leqslant t\right\} \cup\left\{e_{u v} \mid 1 \leqslant u<v \leqslant n\right\} \cup\left\{x_{i} \mid 1 \leqslant i \leqslant n\right\}$,

- $E\left(G_{2}\right)_{1}=\{p q, p r, q r\}$,

- $E\left(G_{2}\right)_{2}=\left\{r a_{i} \mid 1 \leqslant i \leqslant t\right\}$

- $E\left(G_{2}\right)_{3}=\left\{a_{i} e_{u v} \mid u v \in E\left(G_{i}^{c}\right)\right\}$,

- $E\left(G_{2}\right)_{4}=\left\{e_{u v} x_{u}, e_{u v} x_{v} \mid \forall 1 \leqslant u<v \leqslant n\right\}$,

- $E\left(G_{2}\right)=E\left(G_{2}\right)_{1} \cup E\left(G_{2}\right)_{2} \cup E\left(G_{2}\right)_{3} \cup E\left(G_{2}\right)_{4}$.

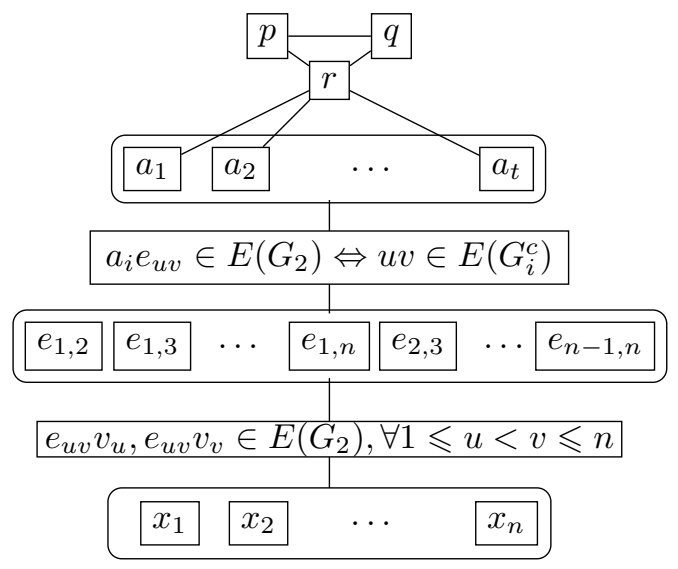

Figure 1: Illustration of the construction of $G_{2}$.

To build $G_{1}$ (see also Figure 2): 
- $V\left(G_{1}\right)=\{p, q, r, a\} \cup\left\{e_{i} \mid 1 \leqslant i \leqslant\left(\begin{array}{l}l \\ 2\end{array}\right)\right\} \cup\left\{x_{i} \mid 1 \leqslant i \leqslant l\right\}$,

- $E\left(G_{1}\right)_{1}=\{p q, p r, q r, r a\}$,

- $E\left(G_{1}\right)_{2}=\left\{a e_{i} \mid 1 \leqslant i \leqslant\left(\begin{array}{l}l \\ 2\end{array}\right)\right\}$,

- $E\left(G_{1}\right)_{3}=\left\{e_{i} x_{u}, e_{i} x_{v} \mid \forall 1 \leqslant i \leqslant\left(\begin{array}{l}l \\ 2\end{array}\right), e_{i}=u v\right\}$,

- $E\left(G_{1}\right)=E\left(G_{1}\right)_{1} \cup E\left(G_{1}\right)_{2} \cup E\left(G_{1}\right)_{3}$.

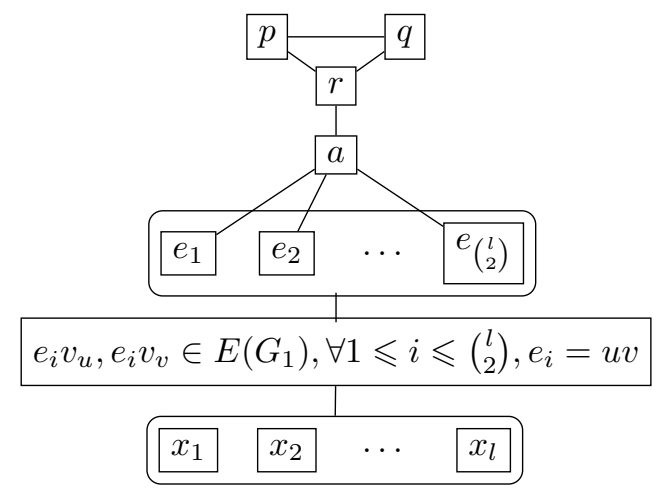

Figure 2: Illustration of the construction of $G_{1}$.

We set $l^{\prime}=\left|V\left(G_{1}\right)\right|$, and $Z=\{p, r\} \cup\left\{e_{u v} \mid 1 \leqslant u<v \leqslant n\right\}$. It is easy to see that $Z$ is indeed a vertex cover for $G_{2}$ and that its size is equal to $\frac{n(n-1)}{2}+2$, which is polynomial in $n$ and hence in the size of the largest instance. Note that the size of the graph $G_{1}$ does not depend on $t$ and is polynomial in $n$, so the size of its vertex cover is also polynomial in $n$ and independent of $t$.

Let us show that $G_{1}$ is an induced subgraph of $G_{2}$ iff at least one of the $G_{i}^{c}$ 's has a clique of size $l$.

$(\Leftarrow)$ Suppose that $G_{i}^{c}$ has a clique of size $l$. We denote by $S \subseteq V\left(G_{i}^{c}\right)$ a clique of size exactly $l$ in $G_{i}^{c}$. We show that there is an induced subgraph $S^{\prime}$ of $G_{2}$ of size $l^{\prime}$, isomorphic to $G_{1}$. We set $V\left(S^{\prime}\right)=\{p, q, r\} \cup\left\{a_{i}\right\} \cup\left\{e_{u v} \mid \forall u v \in E(S)\right\} \cup\left\{x_{u} \mid u \in S\right\}$. One can easily check that this subgraph is isomorphic to $G_{1}$.

$(\Rightarrow)$ Assume now that $G_{1}$ is an induced subgraph of $G_{2}$. Denote by $S^{\prime}$ the subgraph of $G_{2}$ isomorphic to $G_{1}$. Note that the only triangle in $G_{2}$ is pqr. Indeed, $T\left(V\left(G_{2}\right) \backslash\{p\}\right)$ is bipartite. The triangle $p q r$ in $G_{1}$ has therefore to match $p q r$ in $G_{2}$. Moreover, $r$ in $G_{1}$ has to match $r$ in $G_{2}$ since $p$ and $q$ have no edges besides the clique $p q r$. The vertex $a$ in $G_{1}$ can only match a vertex $a_{i}$ for some $i \in\{1, \ldots, t\}$. Then, $e_{1}$ up to $e_{\left(\begin{array}{c}l \\ 2\end{array}\right)}$ in $G_{1}$ has to match $\left(\begin{array}{l}l \\ 2\end{array}\right)$ vertices in $\left\{e_{u v} \mid\right.$ $1 \leqslant u<v \leqslant n\}$ of $G_{2}$ which correspond to actual edges in $G_{i}^{c}$. Finally, $x_{1}$ up to $x_{l}$ in $G_{1}$ has to match $l$ vertices among the $x_{j}$ 's in $G_{2}$. Note that the number of edges in $E\left(G_{1}\right)_{3}$ between the $e_{j}$ 's and the $x_{j}$ 's is exactly $2\left(\begin{array}{l}l \\ 2\end{array}\right)=l(l-1)$. More precisely, each $e_{j}$ touches 2 edges in $E\left(G_{1}\right)_{3}$ and each $x_{j}$ touches $l-1$ edges in $E\left(G_{1}\right)_{3}$. In order to get a match in $G_{2}$, one should find a set of $\left(\begin{array}{l}l \\ 2\end{array}\right)$ edges inducing exactly $l$ vertices. So, this set of $l$ vertices is a clique in $G_{i}^{c}$.

Note that the parameter of MCIS in the previous reduction is exactly the size of $G_{1}$ and the graphs used in the proof are connected. Therefore, we have the following:

Corollary 11. Induced Subgraph Isomorphism and Maximum Common Connected InDUCED SUBGRAPH, parameterized by a bound on the minimum vertex covers of input graphs, do not have a polynomial-size kernel unless $\mathrm{NP} \subseteq$ coNP/poly.

The algorithm of [1] is not single-exponential for parameter sum of the vertex cover numbers. In fact, we show that a single-exponential algorithm is very unlikely. This is, to the best of our knowledge, the first result of this type for parameter vertex cover. 
Theorem 12. Under the ETH, IS $(C) I$ cannot be solved in time $2^{o(k \log k)}$ when parameter $k$ is the sum of the vertex cover number of both graphs.

Proof. We give a reduction from $k \times k$ PeRmutation Clique which linearly preserves the parameter $k$. It is known that this problem does not admit an algorithm with running time $2^{o(k \log k)}$ unless the ETH fails [23]. In the $k \times k$ PERmUtation Clique problem, one is given a graph over the set of vertices $[k] \times[k]$ and the goal is to find a clique of size $k$ such that in each row and in each column exactly one vertex is part of the clique, where a row is the set of vertices $\{(i, 1),(i, 2), \ldots,(i, k)\}$ for some $i \in[k]$, and a column is the set of vertices $\{(1, j),(2, j), \ldots,(k, j)\}$ for some $j \in[k]$.

We first describe how the graph $G_{2}$ is built from any instance $G=([k] \times[k], E)$ of $k \times k$ Permutation Clique. For each row (resp. column) index $i \in[k]$, we add two vertices $r_{i}^{1}$ and $r_{i}^{2}$ (resp. $c_{i}^{1}$ and $c_{i}^{2}$ ) that we link by an edge. For $j \in[2]$, we set $R_{j}=\left\{r_{1}^{j}, r_{2}^{j}, \ldots, r_{k}^{j}\right\}$ (resp. $C_{j}=\left\{c_{1}^{j}, c_{2}^{j}, \ldots, c_{k}^{j}\right\}$ ) and $R=R_{1} \cup R_{2}$ (resp. $C=C_{1} \cup C_{2}$ ). Then, to distinguish row indices from column indices, we add a clique $D_{r}$ of size 6 , and we link one designated vertex $r$ of $D_{r}$ to all the vertices in $R$. We also add a clique $D$ of size 5 with a special vertex $v$ in $D$ such that $v$ is linked to all the vertices in $R_{1} \cup C_{1}$.

Finally, for each edge $e=(i, j)\left(i^{\prime}, j^{\prime}\right)$ of $G$ with $i \neq i^{\prime}$ and $j \neq j^{\prime 2}$, we add a vertex $v(e, 1)$ that we link to the four vertices $r_{i}^{1}, c_{j}^{1}, r_{i^{\prime}}^{2}$, and $c_{j^{\prime}}^{2}$, and a vertex $v(e, 2)$ that we link to the four vertices $r_{i}^{2}, c_{j}^{2}, r_{i^{\prime}}^{1}$, and $c_{j^{\prime}}^{1}$. This ends the construction of $G_{2}$ (see Figure 3 ). The pattern $G_{1}$ depends only on $k$ and is defined as the graph one obtains following the above construction when $G$ have all the edges of the form $(i, i)\left(i^{\prime}, i^{\prime}\right)$ and no other edges (in other words, $G$ has a $k$-clique on the diagonal and nothing else).

Both $G_{1}$ and $G_{2}$ have $R \cup C \cup D_{r} \cup D$ as a vertex cover of size $4 k+11 . G_{2}$ has $|E|+4 k+11=O\left(k^{4}\right)$ vertices and $G_{1}$ has $2\left(\begin{array}{c}k \\ 2\end{array}\right)+4 k+11=O\left(k^{2}\right)$ vertices. To avoid confusion about vertices in $G_{1}$ and $G_{2}$ we will denote the vertices and sets of vertices of $G_{1}$ with a tilde. We now show that the reduction is valid.

Suppose there is a solution $\left\{\left(a_{1}, b_{1}\right), \ldots,\left(a_{k}, b_{k}\right)\right\}$ to the instance of $k \times k$ Permutation Clique. Then, $G_{1}$ is an induced subgraph of $G_{2}$ with the following mapping. We map $\tilde{r}$ to $r$ and $\tilde{v}$ to $v$. We map $\tilde{D}_{r} \backslash\{\tilde{r}\}$ to $D_{r} \backslash\{r\}$ and $\tilde{D} \backslash\{\tilde{v}\}$ to $D \backslash\{v\}$ in an arbitrary way. Then, for each $i \in[k]$ and $j \in[2]$, we map $\tilde{r}_{i}^{j}$ to $r_{a_{i}}^{j}$ and $\tilde{c}_{i}^{j}$ to $r_{b_{i}}^{j}$. We observe that this mapping is one-to-one since $\left(a_{1}, b_{1}\right), \ldots,\left(a_{k}, b_{k}\right)$ is a permutation clique, i.e., $\left\{a_{1}, a_{2}, \ldots, a_{k}\right\}=[k]=\left\{b_{1}, b_{2}, \ldots, b_{k}\right\}$. Finally, for any $j \in[2]$, and any $i \neq i^{\prime} \in[k]$ we map $\tilde{v}(e, j)$ to $v\left(\left(a_{i}, b_{i}\right)\left(a_{i^{\prime}}, b_{i^{\prime}}\right), j\right)$. Note that vertex $v\left(\left(a_{i}, b_{i}\right)\left(a_{i^{\prime}}, b_{i^{\prime}}\right), j\right)$ always exists precisely because $\left\{\left(a_{1}, b_{1}\right), \ldots,\left(a_{k}, b_{k}\right)\right\}$ is a clique.

Conversely, if there is a solution to the IS(C)I instance, we will show that there is a permutation $k$-clique in $G$. There is only one clique of size 6 in $G_{2}$, so the clique $\tilde{D}_{r}$ of size 6 has to be mapped to $D_{r}$. Then, $\tilde{r}$, as the unique vertex of $\tilde{D}_{r}$ of degree larger than 5 , should be mapped to $r$. Now, for the same reasons, $\tilde{D}$ should be mapped to $D$ and $\tilde{v}$ to $v$. Vertices of $\tilde{R}_{1} \cup \tilde{C}_{1}$ are the only $2 k$ unmatched vertices having $\tilde{v}$ as a neighbor, so those vertices should be matched to the only $2 k$ unmatched vertices having $v$ as a neighbor, namely $R_{1} \cup C_{1}$. For similar reasons, $\tilde{R}$ should be mapped to $R$. Now, $\tilde{R}_{2} \cup \tilde{C}_{2}$ can only be mapped to $R_{2} \cup C_{2}$ as the only unmatched vertices having exactly one neighbor in $\tilde{R}_{1} \cup \tilde{C}_{1}\left(R_{1} \cup C_{1}\right)$.

Thus, the $4 k$ vertices of $\tilde{R} \cup \tilde{C}$ can only be mapped to $R \cup C$, such that for $j \in[2], \tilde{R}_{j}$ is mapped to $R_{j}$ and $\tilde{C}_{j}$ is mapped to $C_{j}$. The edges $\tilde{r}_{i}^{1} \tilde{r}_{i}^{2}$ and $r_{i}^{1} r_{i}^{2}$ (resp. $\tilde{c}_{i}^{1} \tilde{c}_{i}^{2}$ and $c_{1}^{1} c_{i}^{2}$ ) further constrains the mapping: if $\tilde{r}_{i}^{1}$ is mapped to $r_{i^{\prime}}^{1}$ then $\tilde{r}_{i}^{2}$ has to be mapped to $r_{i^{\prime}}^{2}$ (resp. if $\tilde{c}_{i}^{1}$ is mapped to $c_{i^{\prime}}^{1}$ then $\tilde{c}_{i}^{2}$ has to be mapped to $c_{i^{\prime}}^{2}$ ). Hence, we can see the mapping from $\tilde{R} \cup \tilde{C}$ to $R \cup C$ as two permutations $\sigma_{r}$ and $\sigma_{c}$ on $k$ elements, such that for $j \in[2]$, for $i \in[k], \tilde{r}_{i}^{j}$ is mapped to $r_{\sigma_{r}(i)}^{j}$ and $\tilde{c}_{i}^{j}$ is mapped to $c_{\sigma_{c}(i)}^{j}$. Then, the current partial mapping can be extended to a solution only if $\left\{\left(\sigma_{r}(1), \sigma_{c}(1)\right), \ldots,\left(\sigma_{r}(k), \sigma_{c}(k)\right)\right\}$ is a clique in $G$. Indeed, $\forall j \in[2], \forall i \neq i^{\prime} \in[k], \tilde{v}\left((i, i)\left(i^{\prime}, i^{\prime}\right), j\right)$ can only be mapped to a potential $v\left(\left(\sigma_{r}(i), \sigma_{c}(i)\right)\left(\sigma_{r}\left(i^{\prime}\right), \sigma_{c}\left(i^{\prime}\right)\right), j\right)$ so that vertex has to exist, meaning that there should be an edge in $G$ between $\left(\sigma_{r}(i), \sigma_{c}(i)\right)$ and $\left(\sigma_{r}\left(i^{\prime}\right), \sigma_{c}\left(i^{\prime}\right)\right)$.

An algorithm solving IS(C)I in time poly $\left(\left|G_{1}\right|,\left|G_{2}\right|\right) 2^{o(k \log k)}$ with $k:=\operatorname{vc}\left(G_{1}\right)+\operatorname{vc}\left(G_{2}\right)$ would therefore translate into an algorithm running in time $2^{o(k \log k)}$ for $k \times k$ PERMUtATION CLIQUe

${ }^{2}$ We ignore the other edges since they are not relevant in finding a permutation clique. 


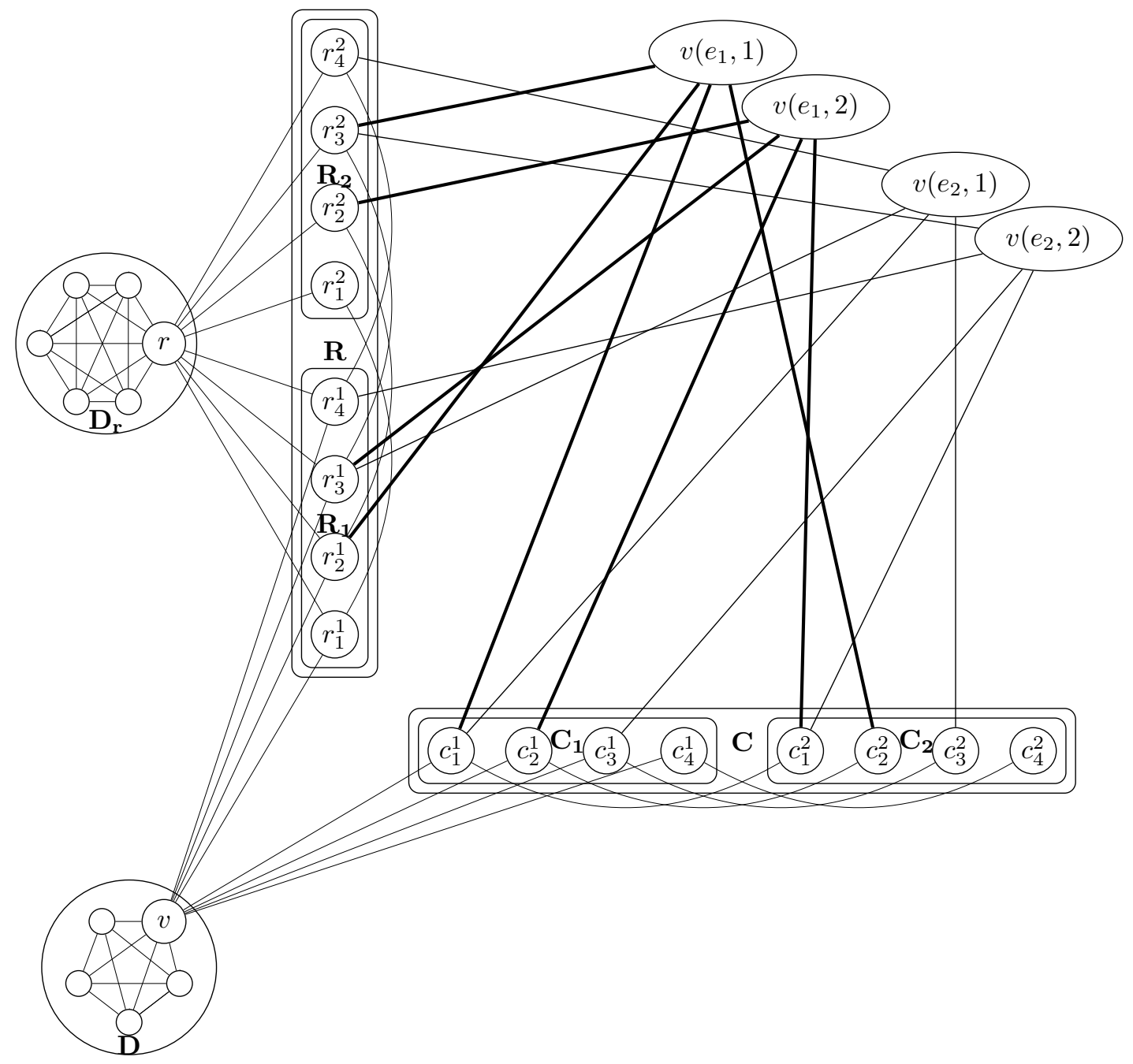

Figure 3: The overall construction of $G_{2}$. We represented only two edges of $G$ : $e_{1}=(2,1)(3,2)$ and $e_{2}=(3,1)(4,3)$. For the sake of readability, the edges encoding $e_{1}$ are enhanced to distinguish them easily from the edges encoding $e_{2}$. 
and contradict the ETH.

Despite the fact that ISI and MCIS have the same parameterized complexity with respect to the natural parameter, they exhibit different behaviors when considering structural parameters. In fact, the latter is paraNP-hard when parameterized by the vertex cover of only one of the two graphs, whereas ISI is FPT when parameterized by the vertex cover of the second (host) graph. To see this, note that when the host graph has a vertex cover of size $k$, the minimum size of a vertex cover in the pattern graph must be bounded by the parameter $k$; otherwise we have a NO-instance. The claim follows from the fixed-parameter tractability of MCIS in this case [1].

Given the negative result of Theorem 9, the next question we pose is whether MCCIS is in FPT with respect to the size of a minimum vertex cover. In [1], a parameterized algorithm is presented for MCIS when the parameter is a bound on the minimum vertex cover number of the input graphs. However, that algorithm cannot help us much for solving MCCIS since it relies on the existence of a feasible solution of size at least $\approx n-k$ which consists of mapping the two big independent sets of the two graphs onto each other. Of course, this is not a feasible solution for MCCIS. In the following we prove that MCCIS is fixed-parameter tractable w.r.t. $k:=\operatorname{vc}\left(G_{1}\right)+\operatorname{vc}\left(G_{2}\right)$.

Theorem 13. Maximum Common Connected Induced Subgraph parameterized by $k:=$ $v c\left(G_{1}\right)+v c\left(G_{2}\right)$ is fixed-parameter tractable.

Proof. In time $O^{*}\left(2^{k}\right)$ (even $O^{*}\left(1.2738^{k}\right)[10]$ ), we can find minimum vertex covers $C_{1}$ and $C_{2}$ in $G_{1}$ and $G_{2}$ respectively. Let $I^{(j)}$ be the independent set $V\left(G_{j}\right) \backslash C_{j}$ for $j \in\{1,2\}$. By assumption, our parameter $k$ is $\max \left(C_{1}, C_{2}\right)$, so we can enumerate all tripartitions of $C_{1}$ and $C_{2}$ in time $O^{*}\left(9^{k}\right)$. We denote by $C_{1, m}, C_{1, u}$ and $C_{1, i}$ (respectively $C_{2, m}, C_{2, u}$ and $C_{2, i}$ ) the three sets of a tripartition of $C_{1}$ (respectively $C_{2}$ ). For $j \in\{1,2\}, C_{j, u}$ corresponds to the vertices of $C_{j}$ that are not matched, so they may be deleted. $C_{j, m}$ comprises the vertices matched to $C_{3-j, m}$ (that is, to the vertex cover of the other graph), and $C_{j, i}$ are the vertices matched to $I^{(3-j)}$, the independent set of the other graph. See Figure 4.

We observe that for $j \in\{1,2\}, I^{(j)}$ can be partitioned into at most $2^{k}$ classes of twins: $I_{1}^{(j)}, I_{2}^{(j)}, \ldots I_{2^{k}}^{(j)}$. A class of twins in this context is a set of vertices with an identical neighborhood in the vertex cover and there are at most $2^{k}$ subsets of $C_{j}$. Potentially, some classes can be empty: they correspond to a subset of the vertex cover $C_{j}$ that is not the (exact) neighborhood of any vertex in $I^{(j)}$.

At this point, we can enumerate the mappings between $C_{1, m}$ and $C_{2, m}$ in time $O^{*}\left(k^{k}\right)$ and the mappings between $C_{j, i}$ and $I^{(3-j)}$ in time $O^{*}\left(\left(2^{k}\right)^{k}\right)=O^{*}\left(2^{k^{2}}\right)$. Indeed, to match a vertex $u$ with a vertex $v$ or a twin of $v$ is equivalent. Thus, in time $O^{*}\left((9 k)^{k} 2^{k^{2}}\right)$ we can enumerate all the solutions of MCIS where only vertices of $I^{(1)}$ could still be matched to vertices of $I^{(2)}$. The optimal map of the independent sets can be done in polynomial time by matching the greatest number of vertices in each equivalent twin class (which is the size of the smaller of the two equivalent twin classes), where a twin class $I_{r}^{(j)}$ in $I^{(j)}$ is equivalent to a twin class $I_{s}^{(3-j)}$ in $I^{(3-j)}$ if the vertices of $N\left(I_{r}^{(j)}\right) \backslash C_{j, u}$ and $N\left(I_{s}^{(3-j)}\right) \backslash C_{3-j, u}$ are in one-to-one correspondence.

To find a solution for MCCIS, the algorithm described in the above proof enumerates all possible maximal common induced subgraphs in time $O^{*}\left((9 k)^{k} 2^{k^{2}}\right)$. The current bottleneck to improve it is when we try to match vertices of the vertex cover with vertices of the independent set. For the not connected version of the problem, a trivial argument can bound the size of the independent set (if this one is big, there is a trivial solution), which cannot be used for the connected version. As such, it can be used as an enumeration algorithm for MCIS.

Corollary 14. Maximum Common Induced Subgraph parameterized by $k:=v c\left(G_{1}\right)+v c\left(G_{2}\right)$ is fixed-parameter enumerable.

Let us finish this section with some general considerations. Note that for ISI, the parameter $\mathrm{vc}+\mathrm{fvs}$ is not the same as fvs $+\mathrm{vc}$. In the latter, the parameter is a bound on the vertex cover of 


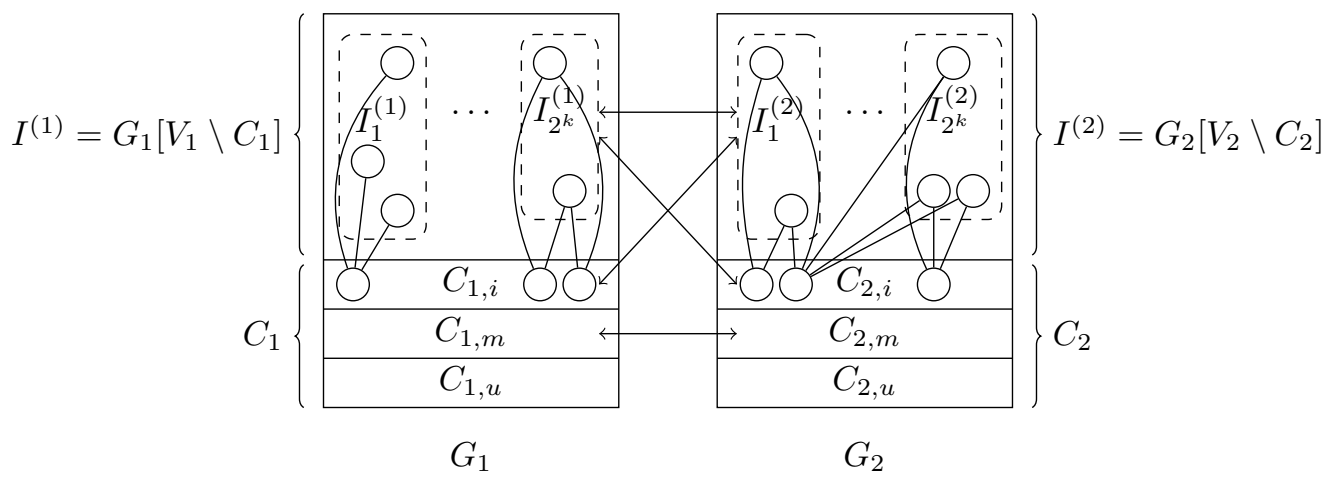

Figure 4: Illustration of the proof of Theorem 13. Dashed boxes represent the classes inside the independent set. Arrows represent the matching between sets of vertices. Sets $C_{1}$ (resp. $C_{2}$ ) represents a vertex cover for $G_{1}$ (resp. $G_{2}$ ).

$G_{2}$ (as well as the feedback vertex set of $G_{1}$ ) which makes ISI in FPT, while it remains open for $\mathrm{vc}+$ fvs. We also note that ISI is not in XP w.r.t. $\mathrm{vc}\left(G_{1}\right)$ by a simple reduction from INDEPENDENT SET: let $G_{2}$ be an edgeless graph on $k$ vertices, then its vertex cover number is 0 .

We now turn our attention to the case where MCIS is parameterized by a combination of the natural parameter and some structural parameter. We note that, in general, such parameterization reduces the problem's complexity. This is most often due to the fixed-parameter tractability of MCIS in $H$-minor free graphs (again, since ISI is FPT in this case [15]). For example, consider the case where the parameter is the sum of some bound $t$ on the feedback vertex set of the input graphs and the natural parameter $k$. The problem is FPT in this case since graphs of $t$-feedback vertex set are $H$-minor free where $H$ is the "fixed" graph consisting of a disjoint union of $t+1$ triangles. The same applies to parameterization by treewidth and the natural parameter by considering $H$ to be the complete graph on $t+2$ vertices, for example.

\section{Conclusion}

We studied the Maximum Common Induced Subgraph and Maximum Common Connected INDUCED SUbGRAPH problems with respect to the solution size on special graph classes such as forests, bipartite graphs, bounded degree graphs, bounded degeneracy graphs, graphs without small (length 3 or 4 ) cycles. The two problems are fixed-parameter tractable on $H$-minor free graphs, which include forests, and bounded degree graphs, but they are W[1]-complete on bipartite graphs of girth 6 and degeneracy 2. This ruled out at the same time two approaches to get fixedparameter algorithms on subclasses of graphs for W-hard problems.

We then considered the use of structural parameters, such as a bound on the minimum vertex covers of the input graphs. Although both MCIS and MCCIS are in FPT in this case, we proved that no kernel of polynomial bound can be obtained unless NP $\subseteq$ coNP/poly and that they cannot be solved in time $2^{o(k \log k)}$ under the ETH. We noted that MCIS is not even in XP with respect to other (smaller) auxiliary parameters, such as treewidth and feedback vertex set. A few open problems remain to be addressed. For example, is MCIS/MCCIS in FPT when parameterized by the combination of the vertex cover number and the feedback vertex set number, or by the vertex cover number and the treewidth? Moreover, it would be interesting to know whether the algorithm for MCCIS of Theorem 13 can be improved to match the lower bound. 


\section{References}

[1] F. N. Abu-Khzam. Maximum common induced subgraph parameterized by vertex cover. Inf. Process. Lett., 114(3):99-103, 2014.

[2] F. N. Abu-Khzam, E. Bonnet, and F. Sikora. On the complexity of various parameterizations of common induced subgraph isomorphism. In J. Kratochvíl, M. Miller, and D. Froncek, editors, Combinatorial Algorithms - 25th International Workshop, IWOCA 2014, volume 8986 of $L N C S$, pages 1-12. Springer, 2014.

[3] T. Akutsu. An RNC algorithm for finding a largest common subtree of two trees. IEICE Transactions on Information and Systems, 75(1):95-101, 1992.

[4] T. Akutsu. A polynomial time algorithm for finding a largest common subgraph of almost trees of bounded degree. IEICE Transactions on Fundamentals of Electronics, Communications and Computer Sciences, 76(9):1488-1493, 1993.

[5] N. Alon and S. Gutner. Linear time algorithms for finding a dominating set of fixed size in degenerated graphs. Algorithmica, 54(4):544-556, 2009.

[6] S. Arumugam, K. R. Chandrasekar, N. Misra, G. Philip, and S. Saurabh. Algorithmic aspects of dominator colorings in graphs. In C. S. Iliopoulos and W. F. Smyth, editors, Combinatorial Algorithms - 22nd International Workshop, IWOCA, volume 7056 of LNCS, pages 19-30. Springer, 2011.

[7] H. L. Bodlaender, B. M. P. Jansen, and S. Kratsch. Kernelization lower bounds by crosscomposition. SIAM J. Discrete Math., 28(1):277-305, 2014.

[8] L. Cai, S. M. Chan, and S. O. Chan. Random Separation: A New Method for Solving FixedCardinality Optimization Problems. In H. L. Bodlaender and M. A. Langston, editors, 2nd International Workshop Parameterized and Exact Computation (IWPEC 2006), volume 4169 of LNCS, pages 239-250. Springer, 2006.

[9] M. Cesati. The turing way to parameterized complexity. J. Comput. Syst. Sci., 67(4):654-685, 2003.

[10] J. Chen, I. A. Kanj, and G. Xia. Improved upper bounds for vertex cover. Theor. Comput. Sci., 411(40-42):3736-3756, Sept. 2010.

[11] P. Damaschke. Induced subgraph isomorphism for cographs is np-complete. In Proc. Workshop on Graph Theoretic Concepts in Computer Science, volume 484 of LNCS, pages 72-78. Springer, 1991.

[12] R. G. Downey and M. R. Fellows. Fundamentals of Parameterized Complexity. Texts in Computer Science. Springer, 2013.

[13] M. R. Fellows, B. M. P. Jansen, and F. A. Rosamond. Towards fully multivariate algorithmics: Parameter ecology and the deconstruction of computational complexity. Eur. J. Comb., $34(3): 541-566,2013$.

[14] M. R. Fellows, B. M. P. Jansen, and F. A. Rosamond. Towards fully multivariate algorithmics: Parameter ecology and the deconstruction of computational complexity. Eur. J. Comb., 34(3):541-566, 2013.

[15] J. Flum and M. Grohe. Fixed-parameter tractability, definability, and model-checking. SIAM Journal on Computing, 31(1):113-145, 2001.

[16] M. R. Garey and D. S. Johnson. Computers and Intractability: A guide to the theory of NP-completeness. W.H. Freeman, San Francisco, 1979. 
[17] H. M. Grindley, P. J. Artymiuk, D. W. Rice, and P. Willett. Identification of tertiary structure resemblance in proteins using a maximal common subgraph isomorphism algorithm. Journal of Molecular Biology, 229(3):707-721, 1993.

[18] M. Grohe, S. Kreutzer, and S. Siebertz. Deciding first-order properties of nowhere dense graphs. In D. B. Shmoys, editor, Symposium on Theory of Computing, STOC 2014, pages 89-98. ACM, 2014.

[19] P. Heggernes, P. van 't Hof, D. Meister, and Y. Villanger. Induced subgraph isomorphism on proper interval and bipartite permutation graphs. Theoretical Computer Science, 562:252 $269,2015$.

[20] R. Impagliazzo, R. Paturi, and F. Zane. Which problems have strongly exponential complexity? Journal of Computer and System Sciences, 63(4):512-530, Dec. 2001.

[21] I. Koch, T. Lengauer, and E. Wanke. An algorithm for finding maximal common subtopologies in a set of protein structures. J. of Comp. Biology, 3(2):289-306, 1996.

[22] D. Lokshtanov, D. Marx, and S. Saurabh. Known algorithms on graphs of bounded treewidth are probably optimal. In Proc. 22nd Annual ACM-SIAM Symposium on Discrete Algorithms, SODA '11, pages 777-789. SIAM, 2011.

[23] D. Lokshtanov, D. Marx, and S. Saurabh. Slightly superexponential parameterized problems. In Proc. 22nd Annual ACM-SIAM Symposium on Discrete Algorithms, SODA '11, pages 760-776. SIAM, 2011.

[24] D. Marx and I. Schlotter. Cleaning interval graphs. Algorithmica, 65(2):275-316, 2013.

[25] J. McGregor and P. Willett. Use of a maximal common subgraph algorithm in the automatic identification of the ostensible bond changes occurring in chemical reactions. J. of Chemical Information and Computer Science, 21:137-140, 1981.

[26] H. Moser and S. Sikdar. The parameterized complexity of the induced matching problem. Discrete Applied Mathematics, 157(4):715-727, 2009.

[27] R. Niedermeier. Invitation to Fixed Parameter Algorithms. Lecture Series in Mathematics and Its Applications. Oxford University Press, 2006.

[28] V. Raman and S. Saurabh. Short cycles make $W$-hard problems hard: FPT algorithms for $W$-hard problems in graphs with no short cycles. Algorithmica, 52(2):203-225, 2008.

[29] J. W. Raymond and P. Willett. Maximum common subgraph isomorphism algorithms for the matching of chemical structures. Journal of Computer-Aided Molecular Design, 16:521-533, 2002.

[30] A. Yamaguchi, K. F. Aoki, and H. Mamitsuka. Finding the maximum common subgraph of a partial $k$-tree and a graph with a polynomially bounded number of spanning trees. Inf. Process. Lett., 92(2):57-63, 2004. 